\title{
Determination and quantification of phthalic acid esters from the soil collected from the municipal solid-waste dumpsite in Maseru using a simple vortex-assisted low-volume organic solvent extraction
}

\begin{abstract}
Phthalic acid esters (PAEs) are some of the most common compounds ubiquitously found in most landfill leachate, owing to their abundance in everyday materials, such as plastic wares, toys, etc., that always find their way into the landfills. This study reports the determination of PAEs from the solid dumpsite in Maseru using a simple low-volume organic solvent agitation extraction of the soil samples. The extraction process included ethylacetate at $0.5 \mathrm{~mL} / \mathrm{g}$ of soil sample, with 20 seconds agitation using a vortex mixer at room temperature. The extraction showed sufficient linearity $\left(\mathrm{R}^{2} \geq 0.9864\right)$ and recoveries ranging from 71 to $120 \%$ for a 17 -component PAE standard mixture. Importantly, three different PAEs (bis-2-ethylhexyl-, octyl- and nonyl- esters respectively) were quantified at $31.74,0.31$ and $0.50 \mu \mathrm{g} / \mathrm{g}$ of soil respectively. The results thus suggest that the dump site does pose some health threat to the Maseru community owing to the fact that it lies upstream of the municipal water sources. However, further work is required to investigate the mobility of these compounds downstream in order to assess the extent of the potential problem. This approach is very viable for limited sample sizes as well as organic solvents.
\end{abstract}

Volume 7 Issue 5 - 2018

\author{
Mosotho J George \\ Department of Chemistry and Chemical Technology, National \\ University of Lesotho, Lesotho
}

Correspondence: Mosotho J George, Department of Chemistry and Chemical Technology, National University of Lesotho, P.O. Roma I80, Lesotho, Tel +266 522I 3502, Fax +266 2234 0000,Email jm.george@nul.ls; maluti2005@gmail.com

Received: June 14, 2018 | Published: September 14, 2018

\section{Introduction}

Waste generation is one of the most unvoidable consequence of human activity. Solid waste generally finds itself in the landfills and other disposal sites where it poses serious threats to aquatic systems and human health. ${ }^{1}$ With the advent of urbanisation, production and filling up of landfills is critical for the Least Developed economies since waste management is still at infancy despite the available knowledge and literature relating to issues of solid waste manangement and health effects thereof. ${ }^{2}$ Interestingly, studies have revealed a link between the economic status and the amount and type of waste generated, with poorer households typically generating less waste with higher organic waste fractions than wealthier counterparts. ${ }^{3}$

Amongst the many chemical compounds monitored from landfills are phthalic acid esters (PAEs) that are believed to have some endocrine activity. ${ }^{4,5}$ Endocrine activity is defined generally as any form on interference with the hormonal system. ${ }^{6}$ The potential health hazards have prompted World Health Organisation to regulate these chemicals in the water bodies below $8 \mu \mathrm{g} / \mathrm{L},{ }^{7}$ and a ban on the use of PAEs in children toys by the EU. ${ }^{8}$ Scaringly, PAEs have been reportedly detected in medical tubings where they are feared to leach into whatever is being infused through the tubes. ${ }^{9}$ One study reported that there was a high occurrence of respiratory ailments in the environment with higher levels of phthalic acid esters than where their levels were lower. ${ }^{10} \mathrm{~A}$ different study correlated the occurrence of premature breasts development in Puerto Rican girls. ${ }^{11}$ Sadly the exposure to PAEs is not easily evaded due to their wide occurrence as additives in almost all plastic household items such as food and pharmaceutical packaging, cosmetic products, vinyl flooring, plastic bags, children's toys, just to mention but a few, where theyare reportedly used for softening hard plastics. ${ }^{12}$

Consequent to a wide spectrum of sources, PAEs are easily released into the environment and can be detectable in many different matrices such as air, water, soils, sewage sludge, just to mention a few. ${ }^{13}$ However, fortunately, they break down quite easily hence they are not persistent. As it has been argued, more waste is normally generated in urban areas as such these compounds are more abundant in the urban area air than in rural areas. ${ }^{14}$ As potential sources, plastic use is regulated in some countries such as South Africa that have introduced some surcharge on the plastic bags in order to curb indiscriminate plastic littering. ${ }^{15}$ This however is still lacking in most other African countries including Lesotho.

The analyses of PAEs from any source seems to be mostly through the use of chromatography and mass spectrometry although there is a potential to use other techniques such as electroanalysis. ${ }^{16}$ These techniques however still require some sample preparation and cleanup before the actual analysis. Soxhlet extraction is one of the most widely effective and affordable technique for extraction of organic compounds from aged soils and sediments prior to the clean-up methods. ${ }^{13}$ This is because it can dissolve the analytes beyond their saturation points since the sample is always extracted with a fresh solvent. However, this approach is being replaced by those that use much less organic solvent and have a potential for pre-concentraiton of the analytes.

Consequently, a number of extraction methods have been reported 
that include both solid-based and liquid-based approaches each with certain merits and demerits. ${ }^{17}$ The traditional liquid-liquid extraction (LLE) has been replaced by the miniaturised techniques that are believed to be faster and more eco-friendly, ${ }^{18}$ these include dispersive liquid-liquid microextraction, ${ }^{19,20}$ drop-based methods ${ }^{21}$ as well as the hollow-fibre protected micro-volume liquids..$^{22}$ Recent reports show a promise in coupling dispersive liquid-liquid microextraction with the hollowfibre protected formats. ${ }^{23,24}$ The solid-based methods include the QuEChERS, ${ }^{25}$ surfactant-modified hydroxide-coated magnetic nanoparticles ${ }^{26}$ molecular imprinted polymers ${ }^{27}$ as well as the solidliquid coupled techniques. ${ }^{28,29}$

This manuscript describes the attempt to use a simple agitationassisted low-volume organic solvent extraction process for extraction and subsequent quantitation of the PAEs from a soil sample from a solidwaste dumpsite. This approach cuts out the aqueous leaching and subsequent extraciton of the analytes as is the case with all the approaches described in the preceding introduction. The quantitation of the analytes was carried out using the standard addition approach, a somewhat under-rated technique despite its effectiveness for analysis of complex matrices as demonstrated recently as it eliminates the somewhat negative matrix effect. ${ }^{30}$

\section{Experimental}

\section{Chemicals and reagents}

A 17-component phthalates mixture (Mix 3: P.I. 08060300) containing benzylbenzoate and the following phthalic acid esters:dimethyl-, diethyl-, diisobutyl-, dibutyl-, dimethylglycol-, bis(4methyl-2-pentyl)-, bisethoxyethyl-, bispentyl-, hexyl-2-ethylhexyl-, benzylbutyl-, bis-hexyl-, bis-n-butoxyethyl-, biscyclohexyl-, bis2ethylhexyl-, bisoctyl, bisnonyl- and bis(7-methyloctyl)- respectively (referred only by their prefices for ease of reference) at $1000 \mu \mathrm{g} / \mathrm{mL}$ each was obtained from Dr Ehrenstorfer GmbH (Augsburg, Germany) in a $1-\mathrm{mL}$ ampule. All solvents used in this study were HPLC and/or GC grade: methanol, toluene,chloroform, dichloromethane, hexane, toluene, were all obtained from Riedel-de Haën (Steinheim Germany). Ethylacetate (AR), was obtained from Associated Chemicals Enterprises (Pty) Ltd (South Africa)

\section{Instrumental methods}

A 7890A Agilent GC-MS fitted with a J\&W HP-5MS column with $30 \mathrm{~m} \times 0.25 \mathrm{~cm} \times 0.25 \mu \mathrm{m}$ dimensions was used. The chromatographic method was developed as follows: Injection port was set at $280^{\circ} \mathrm{C}$; column program: initial temperature of $80^{\circ} \mathrm{C}$ for $4 \mathrm{~min}$, then ramped at $10^{\circ} \mathrm{C} / \mathrm{min}$ to $300^{\circ} \mathrm{C}$ and held for 5 minutes.; the transfer line was set at $280^{\circ} \mathrm{C}$. The carrier gas was UHP Helium at a flow rate of $1 \mathrm{~mL} /$ min through out the analyses. The MS was set on scanning mode, scan range of 50-500amu, for identification of samples, otherwise for quantitative analyses it was set on SIM mode using the respective mass spectra base peak ions of the individual compounds as well as their qualifying ions (Table 1). The temperature settings were $230^{\circ} \mathrm{C}$ for the ion source and $150^{\circ} \mathrm{C}$ for the quadrupole.

\section{Sample collection and storage}

The soil samples were collected in a dump-site at about a spade-depth. Different random samples were collected and mixed appropriately following the coning and quartering approach to enable collection of about $1 \mathrm{~kg}$ of the representative sample. The samples were then air-dried and ground with a pestle and morter. The well- ground sample was sieved through $<1 \mathrm{~mm}$ sieve to achieve an almost uniform particle size. The samples were then stored below $-5^{\circ} \mathrm{C}$ till further use.

\section{Extraction protocol of the phthalates from the soil}

All the extractions, unless otherwise stated, followed this generic approach. About 2-g samples of the soil were spiked with $2 \mathrm{~mL}$ of aqueous $1 \mu \mathrm{g} / \mathrm{mL}$ phthalates and left to equilibrate for 48 hours. The importance of aqueous spiking in the extraction of soil samples has been reported elsewhere. ${ }^{31}$ Thereafter the water (solvent) was evaporated by leaving the samples in the sun until dry to a constant mass a constant mass (about a two weeks). Then to dried soil samples, appropriate volume (as will be detailed in the results section) of the organic solvent were added and extraction took place under different conditions-sonication and agitation. The supernatant solution was pipetted out into $1.8 \mathrm{~mL}$ epperndorf vials and centrifuged to settle the soil particles and obtain a clear supernatant solution. The clear supernatant solution $(2 \mu \mathrm{L})$ was injected into the GC-MS for analysis using the full scan mode. Reference solutions were prepared by spiking about $2 \mathrm{~mL}$ of HPLC grade water with the equal volume of the standard and treated the same way as the soil sample. All the analyses were performed in replicates $(n=3)$ unless specifically stated.

For optimisation of the extraction protocol, a univariate approach was followed to determine optimum conditions for the following parameters: solvent choice, solvent volume, extraction type (sonication vs simple agitation at room temperature) and finally extraction time were evaluated using a $5 \mu \mathrm{g} / \mathrm{mL}$ spiked sample. Thereafter the extraction efficiency of the method was determined combining all the optimised conditions (factors) appropriately using the univariate approach.

\section{Validation of the method}

The method was validated for reproducibility and repeatability using different spiking levels of the PAEs and extracted them a minimum of $n=9$ over 5 days. The linearity of the method was determined by the standard addition approach in the concentration range $100-5000 \mathrm{ng} / \mathrm{mL}(0.1-5 \mu \mathrm{g} / \mathrm{mL})$. The two approaches, namely, $\mathrm{S} / \mathrm{N}$ ratio and statistical, were used and compared for determination of the estimated method LOD and LOQ values respectively. Since the CRMs were not available, no accuracy validation was performed.

\section{Results and discussion}

\section{Optimisation of the separation of the standard mixture of phthalates}

Table 1 shows the compounds, ranked according to their elution order following an injection of a $5 \mu \mathrm{g} / \mathrm{mL}$ methanol solution of the PAEs standard mixture. The table further shows the respective chromatographic properties: retention time, relative molecular mass, the base peak used as reference (used for SIM), the qualifying ion (second in abundance) and the ratio of reference (Ref) ion to qualifying (Qual) ions for identification purposes. As it can be seen, there are only two slight overlaps between the pairs: bis(4-methyl-2-pentyl)-/ bis-ethoxyethyl- with retention times, $\mathrm{t}_{\mathrm{R}}=19.769$ and $19.813 \mathrm{~min}$, respectively, and bis-2-ethylhexyl-/bis-octyl- retention times, 23.587 and $23.691 \mathrm{~min}$ respectively. However these peaks were significantly resolved to be integrated independently. 
Table I Different phthalates with their retention times and mass spectral data

\begin{tabular}{|c|c|c|c|c|c|c|}
\hline & Compound & $\begin{array}{l}\text { Retention } \\
\text { time (min) }\end{array}$ & $\begin{array}{l}\text { RMM } \\
\text { (amu) }\end{array}$ & $\begin{array}{l}\text { Ref. ion } \\
\text { (amu) }\end{array}$ & $\begin{array}{l}\text { Qual. ion } \\
\text { (amu) }\end{array}$ & $\begin{array}{l}\text { SIM/Qual } \\
\text { ratio }\end{array}$ \\
\hline I & dimethyl phthalate & 13.007 & 194 & 163 & 77 & 5.342 \\
\hline 2 & benzylbenzoate & 14.752 & 212 & 105 & 212 & 3.330 \\
\hline 3 & diethyl phthalate & 16.710 & 222 & 149 & 177 & 3.902 \\
\hline 4 & diisobutyl phthalate & 17.748 & 278 & 149 & 223 & 11.352 \\
\hline 5 & dibutyl phthalate & 18.687 & 278 & 149 & 223 & 16.377 \\
\hline 6 & dimethylglycol phthalate & $|9.08|$ & 292 & 59 & 149 & 3.364 \\
\hline 7 & bis-(4-methyl-2-pentyl) phthalate & 19.769 & 334 & 149 & 85 & 2.425 \\
\hline 8 & bis-ethoxyethylphthalate & 19.813 & 310 & 72 & 149 & 1.224 \\
\hline 9 & bis-pentylphthtalate & 20.159 & 306 & 149 & 237 & II.893 \\
\hline 10 & hexyl-2-ethylhexyl phthalate & 20.469 & 334 & 149 & 251 & 10.742 \\
\hline $\mathrm{II}$ & benzylbutyl phthalate & 22.108 & 312 & 149 & 206 & 3.568 \\
\hline 12 & bis-hexyl phthalate & 22.227 & 362 & 149 & 251 & 7.866 \\
\hline 13 & bis-nbutoxyethyl phthalate & 22.922 & 366 & 149 & 193 & 2.871 \\
\hline 14 & biscyclohexyl phthalate & 23.159 & 330 & 149 & 167 & 2.938 \\
\hline 15 & bis(2-ethylhexyl) phthalate & 23.587 & 390 & 149 & 167 & 2.783 \\
\hline 16 & bis-octyl phthalate & 23.691 & 390 & 149 & 279 & 9.000 \\
\hline 17 & bis-nonyl phthalate & 25.061 & 418 & 149 & 293 & 3.842 \\
\hline 18 & bis(7-methyloctyl) phthalate & 25.449 & 418 & 149 & 293 & 3.481 \\
\hline 19 & bis(7-methyloctyl) phthalate & 25.643 & 418 & 149 & 293 & 3.018 \\
\hline
\end{tabular}

Note: RMM indicates the relative molecular mass of the compound.

Amu denotes atomic mass units.

Ref denotes reference ion used for extracted/selected ion monitoring.

Qual denotes the qualifying ion, the second most abundant ion used for confirmation with the reference ion.

Clearly, most PAEs showed a characteristic base peak $\mathrm{m} / \mathrm{z}$ value of 149 which corresponds to the protonated anhydride moiety after the loss of the alkyl chains. This is consistent with the available literature of the study of mass spectrum of phthalates. ${ }^{32}$ Furthermore, the last two compounds, namely, bis(7-methyloctyl) phthalate and bis(7methyloctyl) phthalate were not stated in the mixture information although they were detected. The decreasing peak intensity observed on the chromatogram as the retention time increases demonstrates the limitation of expressing concentration in units of mass as opposed to number of moles which will give due diligence to the abundance of molecules that will be ionized and detected.

\section{Determination of the ideal solvent for soil extractions}

Different 2-g samples of the soil spiked to a concentration of $1 \mu \mathrm{g} /$ $\mathrm{mL}(1 \mu \mathrm{g} / \mathrm{g}$ of soil $)$, allowed to age as decribed in the general procedures, subsequently subjected to extraction using $1 \mathrm{~mL}$ volume of different solvents: methanol, chloroform, dichloromethane, ethylacetate and toluene respectively, followed by agitation using a vortex mixure until all the soil clumps had been broken completely (about 10-15 seconds) into the suspension. Then the generic extraction procedure outlined earlier was followed. Figure 1 presents the relative extraction of the phthalates using different solvents as a percentage of the extraction in methanol using te same volume $(1 \mathrm{~mL})$.

Clearly ethylacetate is the most ideal solvent for this extraction as it gives the highest efficiency for most of the compounds except the higher molecular compounds (bis-octyl-) where toluene seems prefererable. Importantly, the compounds detected in a previous study (bis-2ethyl-hexyl, dibutyl- and di-isobutyl-) seemed to extract better in ethylacetate. ${ }^{33}$ Thus ethylacetate was selected as the ideal solvent to be used in further experiments.

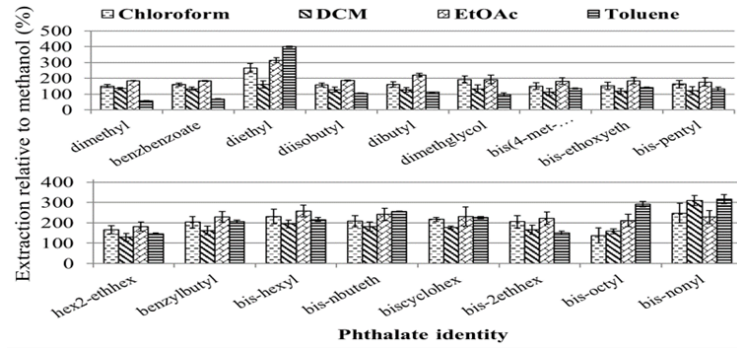

Figure I Extraction of different phthalates from the spiked soil samples using different solvents of the same volume $(V=I m L, n=3)$ - two slides used for clarity.

\section{The effect of solvent volume on extraction efficiency using ethylacetate}

To the treated soil samples, different volumes of the ethylacetate were used to assess the effect of increasing the volume on the extraction of the phthalates from the spiked soil. The results obtained 
from the use of different volume are presented in Figure 2.

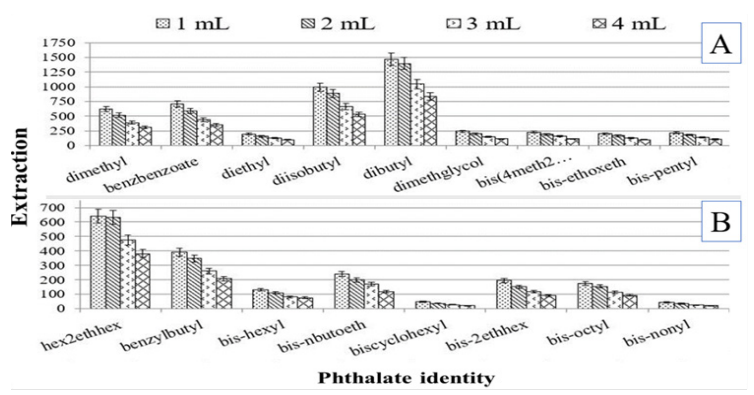

Figure 2 The effect of varying the solvent volume on extraction of the phthalates following a 20 min agitation of spiked soils $(n=3)$. The two slides are used for clarity.

Clearly increasing the volume of the extracting solvent decreases the extraction. This could be due to dilution of the extracted sample. While the smaller volume also risks incomplete extraction as well as the problem of being totally absorbed by the dry soil leaving nothing for further analysis. The $1 \mathrm{~mL}$ volume was just about sufficient and left about $200-300 \mu \mathrm{L}$ (not measured, only estimated by the height in the vial) of the extracting solvent after extraction.

\section{Comparison of sonication and agitation on extraction efficiency}

Sonication is one of the mostly preferred extraction technique for organic compounds bound on soils. However, this is mostly applied on aqueous extractions. To investigate the comparison of the two techniques on extraction efficiency, the pre-treated samples were suspended on equal volumes of ethylacetate and three samples were sonicated for 10 minutes while the other three were agitated for 30 seconds. Agitation could not be prolonged to prevent fatigue since the samples needed to be manually supported on the vortex mixer. Figure 3 shows the comparison of 10 minutes room temperature sonication and 30 seconds room temperature agitation. The net difference of each extraction technique was taken relative to the average obtained using about 15 seconds agitation as in sections 3.2 and 3.3 and plotted against each phthalic acid ester, hence the graph shows a different pattern of responses to the earlier figure where the intensity of the responses decreased down the order.

From Figure 3, it can be seen that the two techniques yielded comparable extraction efficiency. Whether this is because organic solvents were used, or that the samples were not aged sufficiently long, may not be debated much, but it does seem like there is no net gain on using sonication compared to agitation using a simple vortex mixer. Similar observation was made and reported earlier in a work where triazines were extracted from the farm soils using hot-water extraction. ${ }^{29}$ Thus it was decided that since agitation was relatively quicker, and required less sophisticated instrumentation, it was chosen as a best method for the extraction protocol.

\section{Effect of extraction time on the efficiency}

Since the agitation required manual support, it was a bit cumbersome, hence an optimum time was required so that the efficiency would not be compromised by the manual fatigue. Figure 4 shows the effect of extraction time on the efficiency of extraction relative to the 15 seconds agitation using a vortex mixer. eficiency. However this does not apply in general. It could be that in this case it is because the analytes had been spiked into the sample for a very short time. There are reports about the strength of binding of organic compounds in soils which sometimes require much stronger extraction techniques ranging from a simple increased temperature to the supercritical conditions. However, the prolonged agitation also comes at a cost of vaporising the solvent hence decrease the extraction. Figure 3 Comparison of sonication and agitation on extraction of the

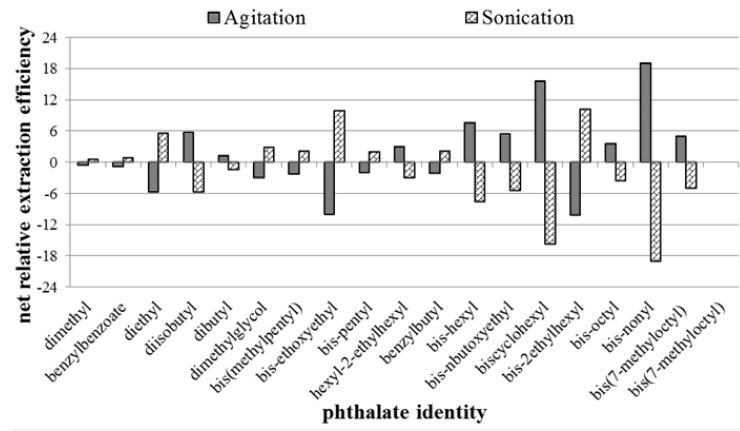

phthalates from the spiked soils using $1 \mathrm{~mL}$ of ethylacetate.

Figure 4 The effect of agitation time on extraction efficiency using ethylacetate.

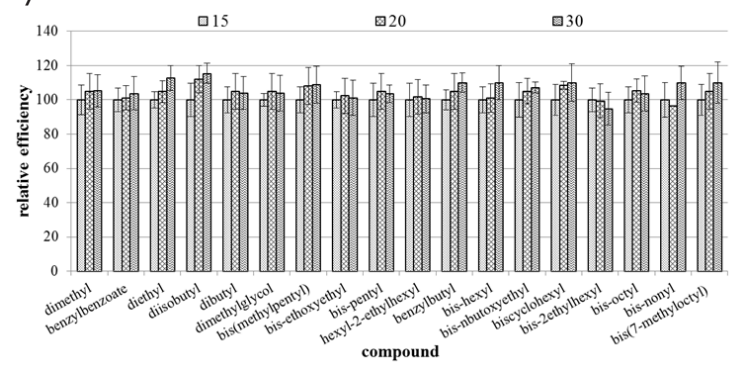

\section{Validation of the method}

The extraction method was validated for reproducibility, repeatability, recovery, linearity and finally the concentration of the detected analytes was determined. The optimised conditions were summarised as follows: $1 \mathrm{~mL}$ of ethylacetate for $2 \mathrm{~g}$ of ground soil sample, agitated for 20 seconds, followed by the injection of $2 \mu \mathrm{L}$ aliquots into the GC-MS.

\section{Validation for reproducibility}

The extraction method was validated for reproducibility and repeatability using different three spiking levels of the PAEs, namely, $0.1,0.5$ and $1.0 \mu \mathrm{g} / \mathrm{mL}$ respectively, and extracted in triplicates $(\mathrm{n}=9)$. Otherwise, the precision obtained throughout the study was also evaluated to assess the reproducibility of the method over 5 days (total number of 45 replicates), with fresh samples being prepared freshly on each day and run on triplicates. Figure 5 shows the reproducibility of the extraction for $0.1 \mu \mathrm{g} / \mathrm{mL}$ spiking level, the lowest of the three spiking levels explored. The reason for the choice of the lowest spiking level was inspired by the fact that as the concentration gets lower, the precision of any method suffers. For ease of reference, the results are residuals of each days' average to the acerage of the total combined replicates $(n=45)$. The residuals are then taken as fractions of the total average.

Clearly, agitation time plays a very limited role in the extraction 


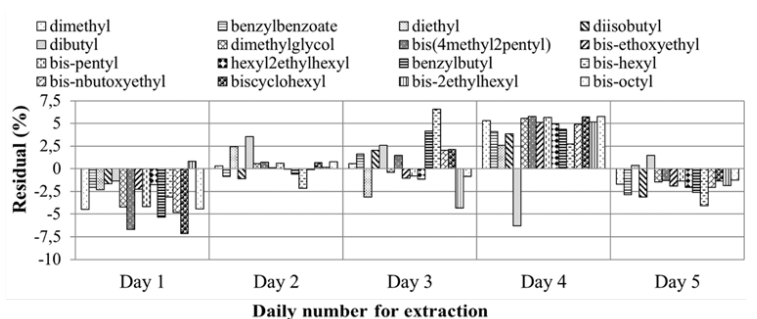

Figure 5 Reproducibility of the extraction protocol at $0.1 \mu \mathrm{g} / \mathrm{mL}$ spiking plotted as residuals of each extraction from the average of each compound over five days showing level over 5 days $(n=45$ given by 3 extractions $\times 3$ suspensions $\times 5$ days).

Since the CRMs were not available, no accuracy validation was performed. As can be seen the results were highly reproducibly with the average relative residuals withing 7.5. There seems to be some intra-day repeatability in that days 1 and 4 resulted in much higher residuals than days 2 and 3 which recorded the lowest values. This could be due to the different laboratory conditions that were not regulaterd such as temperature, given the volatility of ethylacetate. Otherwise the results show significant reproducibility over the fiveday trials.

Recovery, linearity and quantitation of the analytes in the soil samples
To assess the recovery, the soil samples were spiked again at $0.1 \mu \mathrm{g} / \mathrm{mL}$ with the analytical and the surrogates standard and extrated with the optimised conditions against a standard $0.1 \mu \mathrm{g} / \mathrm{mL}$ organic solution used as a reference to calculate the recoveries. The linearity of the extraction was evaluated by standard addition method where a 2-g soil sample was spiked at different levels so as to achieve different concentrations $(100-5000 \mathrm{ng} / \mathrm{mL})$ in the resulting extractions. The combined results as well as the analytical parameters, namely correlation coefficient $\mathrm{R}^{2}$, regression equation, estimated LOD and the concentration of the analytes, obtained from the calibration curves are presented in Table 2. The LOD was estimated using the $\mathrm{S} / \mathrm{N}$ ratio approach.

As can be seen from Table 2, the recovery of most analytes was in the range of $100 \pm 10 \%$ with a few below $90 \%$ (benzylbenzoate, diethyl- and dimethylglycol- at 81,72 and $71 \%$ respectively), while others (bis-n-butoxyethyl-, bis-cyclohexyl-, bis-2-ethylhexyl-, bisoctyl- and bis-nonyl-) range between 125 and $160 \%$. Importantly these included bis-2-ethylhexylphthalate which was detected in the soil, hence the increase in extraction efficiency could be attributed to their presence in the soil sample in addition to the spiked amount. Another issue of interest is the negative y-intercept observed with the two compounds - bis-octyl and bis-nonyl, although not recorded, these two recoveries showed very high standard deviations owing to their low response factors (Figure 1) (Figure 2). The last two compounds were not detected at the concentration range of $0.1 \mu \mathrm{g} / \mathrm{mL}$, hence the recovery entry is empty.

Table 2 Some analytical data obtained from the calibration and regression analysis

\begin{tabular}{|c|c|c|c|c|}
\hline Compound & Recovery (\%) & Linearity $\mathbf{R}^{2}$ & LOD*( $\mu \mathrm{g} / \mathrm{mL})$ & Conc.in soil \# ( $\mu \mathrm{g} / \mathrm{g}$ of soil) \\
\hline dimethyl & 90 & 0.9975 & 0.01 & N.D. \\
\hline benzylbenzoate & 81 & 0.9974 & $<0.01$ & N.D. \\
\hline diethyl & 72 & 0.9983 & $<0.01$ & N.D. \\
\hline diisobutyl & 89 & 0.9993 & $<0.01$ & N.D. \\
\hline dibutyl & 97 & 0.9973 & $<0.01$ & N.D. \\
\hline dimethylglycol & 71 & 0.9864 & 0.01 & N.D. \\
\hline bis(4methyl2pentyl) & 101 & 0.9866 & 0.01 & N.D. \\
\hline bis-ethoxyethyl & 97 & 0.9985 & 0.01 & N.D. \\
\hline bis-pentyl & 91 & 0.9908 & 0.01 & N.D. \\
\hline hexyl-2-ethylhexyl & 96 & 0.9988 & 0.01 & N.D. \\
\hline benzylbutyl & 110 & 0.9992 & 0.01 & N.D. \\
\hline bis-hexyl & 103 & 0.9934 & 0.05 & N.D. \\
\hline bis-nbutoxyethyl & 123 & 0.9972 & 0.05 & N.D. \\
\hline biscyclohexyl & 114 & 0.9972 & 0.05 & N.D. \\
\hline bis(2ethylhexyl) & 125 & 0.9988 & 0.05 & 31.74 \\
\hline bis-octyl & 143 & 0.9972 & 0.10 & 0.61 \\
\hline bis-nonyl & 163 & 0.9965 & 0.10 & 1.01 \\
\hline bis(7-methyloctyl) & - & 0.9953 & $>0.10$ & N.D. \\
\hline bis(7-methyloctyl) & - & 0.9969 & $>0.10$ & N.D. \\
\hline
\end{tabular}

*LOD determined by the $\mathrm{S} / \mathrm{N}$ ratio approach.

\#Concentration in the soil determined by the standard addition method, with N.D. denoting that the analyte was not detectable.

The data demonstrated sufficient linearity with correlation coefficient higher than 0.9864 (dimethylglycol- ester). The LOD determined by the calibration method yielded $8.28 \mathrm{ng} / \mathrm{mL}$ (about $0.008 \mu \mathrm{g} / \mathrm{mL}$ ) slightly lower than that obtained using the $\mathrm{S} / \mathrm{N}$ approach $(0.01 \mu \mathrm{g} / \mathrm{mL}$, Table 2$)$, for bis-2-ethylhexyl-ester, which is the most important analyte in this study. This is inconsistent with the reported literature on determination of LODs from chromatographic analysis. ${ }^{34}$ The LOD obtained using $\mathrm{S} / \mathrm{N}$ ratio was observed to be lower than that obtianed from the statisical methods. The LODs for other compounds estimated from $\mathrm{S} / \mathrm{N}$ ratio were in the range $0.01-0.05 \mu \mathrm{g} / \mathrm{g}$ of soil depending on the detectablity of the compound. Since there is no pre-concentration in this approach, the observed detection limits are determined primarily based on the instrumental sensitivity.

Regarding the quantification aspects, the concentration of the bis-2-ethylhexylphthalate in the extract was found to be $15.87 \mu \mathrm{g} /$ $\mathrm{mL}$ which translates $31.37 \mu \mathrm{g} / \mathrm{g}$ of soil sample used since $1 \mathrm{~mL}$ of methanol was used to extract $2 \mathrm{~g}$ of soil sample. The other compounds that were quantified include nonyl and octyl esters which were determined to be 0.50 and $0.31 \mu \mathrm{g} / \mathrm{mL}$ (about 1.00 and $0.62 \mu \mathrm{g} / \mathrm{g}$ of 
soil sample respectively)

\section{Conclusion}

This study has demonstrated that a simple agitation with a lowvolume solvent could achieve considerable recovery of the analytes. This is highly appreciated since most to the laboratories do not have sonication and/or Soxhlet apparatus, but agitation can even be practised with a simple shaking. The other two important aspects that are demonstrated are: 1) the potential to by-pass the usual preparation of aqueous solutions prior to sample extraction and clean-up, thus reducing the number of steps in the analysis; 2) the low sample amount required for the analysis compared to classical Soxhlet extraction. The importance of volume in the pre-concentration has also been demonstratted by the dilution observed using larger volumes. This thus confirms the observed enrichment in the microextraction approach where much smaller volumes $(1-10 \mu \mathrm{L})$ are used for preconcentration of the analytes.

Besides the commonly studied analytical parameters such as recovery, linearity, reproducibility and limits of detection, that are all satisfactory, the quantification of the detected analytes was performed with bis(2-ethylhexyl-) ester being the highest at $31.37 \mu \mathrm{g} / \mathrm{g}$ of soil. This is attributable to the wide application of this chemical in most commonly used plastics. The other two long-chain aliphatic esters, nonyl- and octyl- were at much lower concentration $(0.5-1 \mu \mathrm{g} / \mathrm{g}$ of soil). While the approach does not add much novelty in the sample preparation tehcniques as alluded to, it presented some valuable information on the presence and levels of these compounds in this dump site. As such it affords a basis for tracking the mobility of these compounds downstream following heavy rains, whether by seepage or surface washed-off with the bound soil. It also adds a significant value to the environmental health considering a variety of human activity including fishing taking place downstream of this dumpsite.

\section{Acknowledgements}

The National University of Lesotho (RCC Grant No. P116-9008) is gratefully acknowledged for the financial support in carrying out this work.

\section{Conflict of interest}

The author declares no conflict of interest regarding the publication of this manuscript.

\section{References}

1. T Eggen, M Moeder, A Arukwe. Municipal landfill leachates: a significant source for new and emerging pollutants. Science of the Total Environment. 2012;408:5147-5157.

2. EA Udofia, JN Fobil, G Gulis. Solid medical waste management in Africa. African Journal of Environmental Science and Technology. 2015;9(3):244-254.

3. Y Vögeli, CR Lohri, A Gallardo, et al. Anaerobic Digestion of Biowaste in Developing Countries: Practical Information and Case Studies. Swiss Federal Institute of Aquatic Science and Technology (Eawag), Dübendorf, Switzerland; 2014.

4. MA Kamrin. Phthalate risks, phthalate regulation, and public health: a review. Journal of Toxicology and Environmental Health. Part B. 2009;12(2)157-174.

5. O Albertz, B Jegou. A critical assessment of the endocrine susceptibility of the human testis to phthalates from fetal life to adulthood. Human
Reproduction Update. 2013;20(2):231-249.

6. RT Zoeller, TR Brown, LL Doan, et al. Endocrine-Disrupting Chemicals and Public Health Protection: A Statement of Principles from The Endocrine Society. Endocrinology. 2012;153(9):4097-4110.

7. Guidelines for Drinking-water Quality. World Health Organization. 3rd edition. Geneva: Switzerland; 2004.

8. Ban of phthalates in childcare articles and toys. European Commission. Belgium; 1999.

9. S Sathyanarayana. Phthalates and children's health. Curr Probl Pediatr Adolesc Health Care. 2008;38(2):34-39.

10. B Kolarik, C Bornehag, K Naydenov, et al. The concentration of phthalates in settled dust in Bulgarian homes in relation to building characteristic and cleaning habits in the family. Atmospheric Environment. 2008;42(37):8553-8559.

11. I Colón, D Caro, CJ Bourdony, et al. Identification of phthalate esters in the serum of young Puerto Rican girls with premature breast development. Environmental Health Perspectives. 2000;108(9):895900.

12. IHS Chemical. Chemical Economics Handbook, Plasticizers; 2015.

13. S Net, A Delmont, R Sempéré, et al. Reliable quantification of phthalates in environmental matrices (air, water, sludge, sediment and soil): A review. Science of the Total Environment. 2015;515:162-180.

14. R Rudel, L Perovich. Endocrine disrupting chemicals in indoor and outdoor air. Atmos Environ (1994). 2008;43(1):170-181.

15. The plastic bag agreement. Ministry of Environment Affairs and Tourism. The Environmark, Pretoria, South Africa; 2002.

16. MS Qureshi, ARBM Yusoff, MDH Wirzal, et al. Methods for the determination of endocrine disrupting phthalate esters. Critical Reviews in Analytical Chemistry. 2016;46(2):146-159.

17. I Jiménez-Díaz, F Vela-Soria, R Rodríguez-Gómez, et al. Analytical methods for the assessment of endocrine disrupting chemical exposure during human fetal and lactation stages: A review. Analytica Chimica Acta. 2015;892:27-48.

18. MA Farajzadeh, SR Aghdam, MRA Mogaddam, et al. An elevated temperature-dispersive liquid-liquid microextraction method combined with GC-flame ionization detection as a sensitive method for determining phthalate esters. Analytical Methods. 2015;7(10):42694277.

19. P Viñas, N Campillo, M Pastor-Belda, et al. Determination of phthalate esters in cleaning and personal care products by dispersive liquidliquid microextraction and liquid chromatography-tandem mass spectrometry. Journal of Chromatography A. 2015;1376:18-25.

20. MA Farajzadeh, SM Sorouraddin, MRA Mogaddam. Microextraction methods for the determination of phthalate esters in liquid samples: A review. Journal of Separation Science. 2015;38(14):2470-2487.

21. A Qi, M Li, L Mao. Application of single drop microextraction in the determination of phthalate esters and parabens in drugs by gas chromatography-mass spectrometry. Se pu. 2008;26(3):306-309.

22. J Li, Q Su, KY Li, et al. Rapid analysis of phthalates in beverage and alcoholic samples by multi-walled carbon nanotubes/silica reinforced hollow fibre-solid phase microextraction. Food Chemistry. 2013;141(4):3714-3720.

23. T Letseka, MJ George. Towards coupling dispersive liquid-liquid microextraction with hollow fibre liquid phase microextraction for extraction of organic pollutants of agricultural origin. Analytical Chemistry Research. 2016;10:28-32.

24. T Letseka, MJ George. Hollow-Fibre-Supported Dispersive Liquid- 
Liquid Microextraction for Determination of Atrazine and Triclosan in Aqueous Samples. International Journal of Analytical Chemistry. 2017:1-8

25. P Yin, $\mathrm{X}$ Liu, $\mathrm{H}$ Chen, et al. Determination of 16 phthalate esters in tea samples using a modified QuEChERS sample preparation method combined with GC-MS/MS. Food Addit Contam Part A Chem Anal Control Expo Risk Assess. 2014;31(8):1406-1413.

26. X Zhao, S Liu, P Wang, et al. Surfactant-modified flowerlike layered double hydroxide-coated magnetic nanoparticles for preconcentration of phthalate esters from environmental water samples. Journal of Chromatography A. 2015;1414:22-30.

27. J Qiao, M Wang, H Yan, et al. Dispersive solid-phase extraction based on magnetic dummy molecularly imprinted microspheres for selective screening of phthalates in plastic bottled beverages. Journal of Agriculture and Food Chemistry. 2014;62(13):2782-2789.

28. X Zhang, W Yang, N Wang, et al. Synthesis and Evaluation of Ionic Liquid-Mediated Molecularly Imprinted Polymer for Highly Selective Recognition of Dibutyl Phthalate from Liquor Samples. Advances in Polymer Technology. 2017;36(2):220-229.

29. MJ George, L Marjanovic, DBG Williams. Solvent-assisted headspace sampling using solid phase microextraction for the analysis of phenols in water. Analytical Chemistry. 2015;87(19):9559-9562.

30. MJ George. Application of the mixed-solvent BID-SDME technique for determination of some stilbene hormones in water downstream of a cattle slaughterhouse, using gas chromatography and mass spectrometry. International Journal of Environmental Analytical Chemistry. 2016;96(3):247-256.

31. DBG Williams, MJ George, L Marjanovic. Rapid detection of atrazine and metolachlor in farm soils: gas chromatography-mass spectrometry based analysis using the bubble-in-drop single drop micro-extraction (BID-SDME) enrichment method. Journal of Agriculture and Food Chemistry. 2014;62(31):7676-7681.

32. RA Hites. Phthalates in the Charles and the Merrimack Rivers Environmental Health Perspectives. 1973;3:17-21.

33. MJ George. Analysis of endocrine disrupting chemicals from the solid waste dumpsite in Maseru using Gas Chromatography and Mass Spectrometry. A report to OPCW. The Hague; Netherlands; 2015.

34. DBG Williams, MJ George, R Meyer, et al. Bubbles in Solvent MicroExtraction: The Influence of Intentionally Introduced Bubbles on Extraction Efficiency. Analytical Chemistry. 2011;83:6713-6716. 